\title{
Critical Hamiltonians with long range hopping
}

\section{S. Levitov}

Physics Department, Center for Materials Sciences \& Engineering, Massachusetts Institute of Technology, 77 Massachusetts Ave., Cambridge, MA 02139, USA

levitov@mit.edu

Received 29 July 1999, accepted dd.mm.yyyy by ue

Abstract. Critical states are studied by a real space RG in the problem with strong diagonal disorder and long range power law hopping. The RG flow of the distribution of coupling parameters is characterized by a family of non-trivial fix points. We consider the RG flow of the distribution of participation ratios of eigenstates. Scaling of participation ratios is sensitive to the nature of the RG fix point. For some fix points, scaling of participation ratios is characterized by a distribution of exponents, rather than by a single exponent.

The RG method can be generalized to treat certain fermionic Hamiltonians with disorder and long range hopping. We derive the RG for a model of interacting two-level systems. Besides couplings, in this problem the RG includes the density of states. The density of states is renormalized so that it develops a singularity near zero energy.

Keywords: localization, scaling, multifractality

PACS: $72.15 . \mathrm{Rn}, 72.20 . \mathrm{Ee}, 73.20 . \mathrm{Fz}$

Conventionally, localization is studied for Hamiltonians with matrix elements rapidly decreasing as a function of the distance. For instance, the canonical Anderson model is defined on a lattice so that disorder is diagonal and hopping occurs between neighboring sites. In this model the localization transition is reached by reducing the amplitude of hopping $V$ below the threshold $V_{c}$ approximately given by the disorder bandwidth $W$ divided by the number of nearest neighbors.

The situation is completely different for the problem with long range hopping[1]. In a $d$-dimensional space, for hopping amplitude falling as some power of the distance, $V\left(r-r^{\prime}\right) \sim\left|r-r^{\prime}\right|^{-\alpha}$, all states are extended for $\alpha \leq d$ for both strong and weak disorder. At the same time, for $\alpha>d$ and strong disorder the states are localized.

Thus, one can study localization transition by varying the exponent $\alpha$ at fixed disorder strength [2, 3, 因, 可]. This transition is more tractable than the conventional one because of the availability of a small parameter given by the ratio of hopping to disorder strength [2, 3]. At the transition line $\alpha=d$, a real space renormalization group (RG) can be constructed for the distribution of couplings. The RG flow obeys certain conservation laws, and, depending on the details of the Hamiltonian, the flow may or may not have a nontrivial fix point.

Recently, this problem reappeared in the context of quantum chaos in Kepler billiards [3]. The RG approach was used in [3] to treat scaling of wave functions at the transition. In Sec. 2 below the RG scheme is applied to study the distribution of participation ratios. We find that, depending on the type of the fix point, participation ratios are either characterized by a single scaling exponent, or by a distribution 
of scaling exponents.

One can extend the RG method [2, 3] to certain many-body systems with disorder and long range interactions. One interesting example is the quantum problem of two level systems with long range coupling [8, 7, 9]. In Sec. 通 we develop an RG for a $d=3$ problem with couplings falling as a cube of the distance. From the RG flow derived for the distribution of the wavefunctions and for the density of states we find that:

(i) Wavefunctions of all excitations are delocalized;

(ii) Under RG of the density of states a singularity develops near zero energy due to states with small energy condensing into a peak. We discuss the relation of this new state of interacting two level systems to the Efros-Shklovskii Coulomb gap state [7].

\section{Renormalization group}

We consider an ensemble of Hamiltonians on a $d$-dimensional lattice:

$$
E \psi_{\mathbf{r}}=E_{\mathbf{r}} \psi_{\mathbf{r}}+\sum_{\mathbf{r}^{\prime} \neq \mathbf{r}} V_{\mathbf{r}, \mathbf{r}^{\prime}} \psi_{\mathbf{r}^{\prime}}, \quad \text { where } \quad V_{\mathbf{r}, \mathbf{r}^{\prime}}=\frac{\vec{a}_{\mathbf{r}} \cdot \vec{a}_{\mathbf{r}^{\prime}}}{\left|\mathbf{r}-\mathbf{r}^{\prime}\right|^{d}}
$$

Here $E_{\mathbf{r}}$ are random numbers, and $\vec{a}_{\mathbf{r}}$ are random $n$-component vectors. The distribution of $E_{\mathbf{r}}$ is uniform with density $\nu: d N / d E=\nu$ for $|E|<1 / 2 \nu ; 0$ for $|E|>1 / 2 \nu$. We assume that the distribution of $\vec{a}_{\mathbf{r}}, d P=f(\vec{a}) d^{n} a$, is such that all moments of $f(\vec{a})$ exist. Otherwise, the exact form of $f(\vec{a})$ is unimportant.

The coupling strength is characterized by dimensionless parameter $\lambda=\left\langle a_{\mathbf{r}}^{2}\right\rangle n \nu$, where $n$ is the density of lattice sites. In the weak coupling regime, $\lambda \ll 1$, a real space RG flow of the Hamiltonians of the form (11) can be derived. This is done by considering resonance pairs and arguing that they correctly account for the flow of the coupling distribution to the leading logarithmic order (see [2, 3]).

The RG flow preserves the form of the hopping amplitudes $V_{\mathbf{r}, \mathbf{r}^{\prime}}$ in (11), and modifies the distribution $f(\vec{a})$. The distribution of energies $E_{\mathbf{r}}$ is renormalized near the tails [6], but remains constant in the middle part. In the middle of the spectrum, $\left|E_{\mathbf{r}}\right| \ll \nu^{-1}$, one can treat $E_{\mathbf{r}}$ as quasirandom numbers with fixed uniform distribution.

To introduce some objects appearing in the RG equation, let us explicitly diagonalize the problem of a resonance between two sites $\mathbf{r}_{\mathbf{1}}$ and $\mathbf{r}_{\mathbf{2}}$ :

$$
E \psi_{1}=E_{1} \psi_{1}+V_{\mathbf{r}_{1}, \mathbf{r}_{2}} \psi_{2}, \quad E \psi_{2}=E_{2} \psi_{2}+V_{\mathbf{r}_{2}, \mathbf{r}_{1}} \psi_{1} .
$$

Two eigenstates $\psi^{+}$and $\psi^{-}$are defined by

$$
\psi^{+}=\cos \theta \psi_{1}+\sin \theta \psi_{2}, \quad \psi^{-}=-\sin \theta \psi_{1}+\cos \theta \psi_{2},
$$

with

$$
\tan \theta=\sqrt{\tau^{2}+1}-\tau, \quad \tau=\frac{E_{1}-E_{2}}{2 V_{\mathbf{r}_{1}, \mathbf{r}_{2}}} .
$$

The energies of the states (位) are

$$
E_{ \pm}=\frac{1}{2}\left(E_{1}+E_{2} \pm \sqrt{\left(E_{1}-E_{2}\right)^{2}+4 V_{\mathbf{r}_{1}, \mathbf{r}_{2}}^{2}}\right)
$$


The transformation rule for the vectors $\vec{a}_{1,2}$ follows from the relation

$$
\vec{a}_{1} \psi_{1}+\vec{a}_{2} \psi_{2}=\vec{a}_{+} \psi^{+}+\vec{a}_{-} \psi^{-} .
$$

One obtains

$$
\vec{a}_{+}=\cos \theta \vec{a}_{1}+\sin \theta \vec{a}_{2}, \quad \vec{a}_{-}=-\sin \theta \vec{a}_{1}+\cos \theta \vec{a}_{2}
$$

The role of transformed $\vec{a}_{ \pm}$is that they determine hopping matrix elements $V_{\mathbf{r}, \mathbf{r}_{ \pm}}=$ $\vec{a}_{\mathbf{r}} \cdot \vec{a}_{ \pm} /\left|\mathbf{r}-\mathbf{r}_{ \pm}\right|^{d}$ for all remote sites $\left|\mathbf{r}-\mathbf{r}_{ \pm}\right| \gg\left|\mathbf{r}_{\mathbf{1}}-\mathbf{r}_{\mathbf{2}}\right|$, where $\mathbf{r}_{ \pm}=\left(\mathbf{r}_{\mathbf{1}}+\mathbf{r}_{\mathbf{2}}\right) / 2$.

Subsequently diagonalizing pair resonances at all distances, from small to large, one derives [2] an RG equation for the distribution of $\vec{a}_{\mathbf{r}}$ 's. It has a form of a first order differential equation with respect to the RG time $t=\ln (L)$ with an integral operator on the right hand side:

$$
\begin{aligned}
\frac{\partial}{\partial t} f(a) & =2 A_{\mathrm{d}} \nu n \int d \tau d^{n} a_{1} d^{n} a_{2}\left|\vec{a}_{1} \cdot \vec{a}_{2}\right| f\left(a_{1}\right) f\left(a_{2}\right) \\
& \times\left[\delta\left(a-a_{+}\right)+\delta\left(a-a_{-}\right)-\delta\left(a-a_{1}\right)-\delta\left(a-a_{2}\right)\right] .
\end{aligned}
$$

Here $A_{\mathrm{d}}$, the area of a $d$-dimensional sphere, arises from $d V=A_{\mathrm{d}} r^{d-1} d r$.

The flow of the distribution $f(a, t)$ obtained by solving (8) has a number of properties similar to that of Boltzmann kinetic equation. The reason is that in considering only pair resonances and ignoring correlations between resonances at different scales one makes an approximation of the same nature as the pair collision approximation in Boltzmann theory of weakly nonideal gases.

Two properties of the flow (8) are of interest. First, all second moments

$$
G_{\alpha \beta}=\left\langle a_{\alpha} a_{\beta}\right\rangle=\int f(a) a_{\alpha} a_{\beta} d^{n} a
$$

are conserved. Second, one can define entropy

$$
H[f]=-\int \ln (|a| f(a)) f(a) d^{n} a
$$

and, for the flow (8) modified by $\left|\vec{a}_{1} \cdot \vec{a}_{2}\right| \rightarrow\left|\vec{a}_{1}\right|\left|\vec{a}_{2}\right|$, prove an H-theorem, $\partial H / \partial t \geq 0$.

These results can be used to fully characterize the fix points of the flow (8). By maximizing the entropy (10) for given moments $G_{\alpha \beta}$ one obtains the family

$$
f_{G}(a)=(A /|a|) \exp \left(-a_{\alpha} G_{\alpha \beta} a_{\beta}\right) .
$$

The normalization constant $A$ depends on the matrix $G$.

It is interesting that the distributions (11) are normalizable only when the number of components $n$ is greater than one (and matrix $G_{\alpha \beta}$ is nondegenerate). Because of that, the flow (8) has very different properties for $n=1$ and $n>1$.

In the $n>1$ case, for any initial conditions $f(a)_{t=0}$, the distribution $f(a)$ evolves to one of stationary distributions (11). On the other hand, for $n=1$ possible fix point distributions allowed by $\mathrm{H}$-theorem are non-normalizable. Therefore, since $\int f(a)_{t=0} d a=1$, the flow (8) does not have a fix point.

These two qualitatively different types of flow are displayed in Figures 1,2. The simulation was performed by using $N=10^{4}$ states characterized by random energies and coupling vectors. The energy distribution was uniform with $\nu=1$ and did not evolve. The distribution of $\vec{a}$ evolved to a fix point of the form (11) for $n=2$ and diverged for $n=1$. 


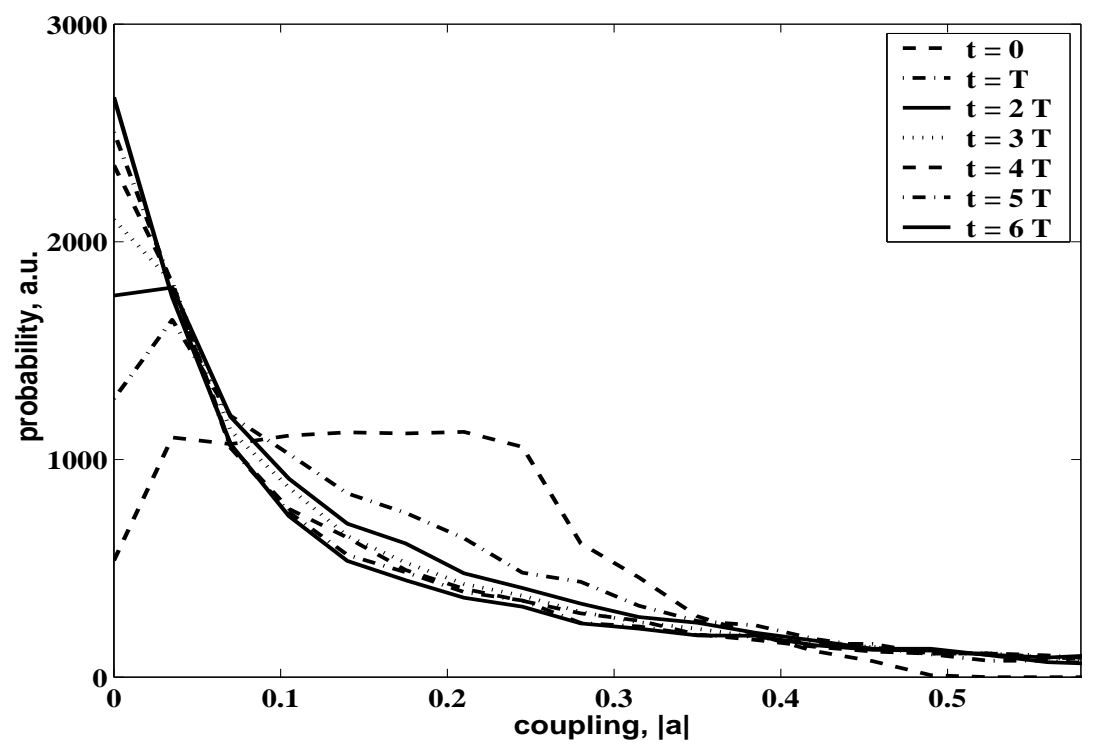

Fig. 1 The flow ( 8 ) for $n=2$. Initial distribution is uniform in the disk $a_{1}^{2}+a_{2}^{2}<r_{0}^{2}$, and hence $f(a)$ is circular symmetric. At large times the distribution approaches a fix point of the form (11) Parameters used in this simulation are: $\nu=1, r_{0}=0.6, T=4$. Dimensionless coupling $\lambda=0.2$ for these parameters.

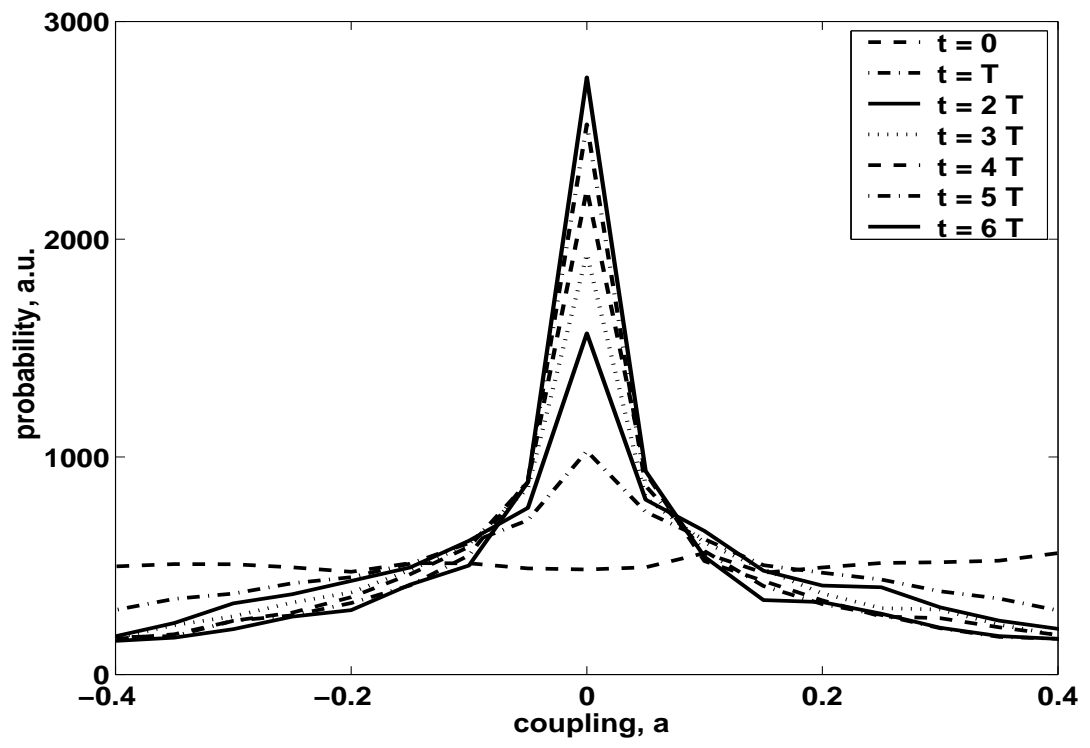

Fig. 2 The flow (8) for $n=1$. Initial distribution is uniform in the interval $|a|<r_{0}$. At large times the distribution looks more and more like $(A /|a|) e^{-g a^{2}}$ with time-dependent $A$ and $g$. Note the divergence developing near $a=0$ and in the tails. Parameters used in this simulation are: $\nu=1, r_{0}=0.5, T=4$. Dimensionless coupling $\lambda=0.08$. 


\section{Scaling of wavefunctions}

The RG hierarchy of states constructed as resonances on different scales implies fractal structure the wavefunctions. To understand scaling of eigenstates, one can extend RG by including in it the distribution of wavefunction amplitudes.

Below we consider the distribution of participation ratios of the states. For a normalized state $\psi_{\mathbf{r}}^{(i)}$ its participation ratio $p_{4}$ is defined as $p_{4}=\sum_{\mathbf{r}}\left|\psi_{\mathbf{r}}^{(i)}\right|^{4}$ In the localization problem the participation ratios are used as a measure of the degree of localization. The scaling exponent $\mu$ of participation ratios defined as $p \sim L^{-\mu}$, where $L$ is the system size, characterizes possible universality classes. In the localized regime $\mu=0$, in the delocalized regime $\mu=d$, and at the critical point $0<\mu<d$.

It is straightforward to put the distribution of participation ratios in the RG scheme. For that one has to consider the flow of the distribution $f(a, p)$. Following the discussion of Sec. 1, one finds the participation ratios $p_{ \pm}$of the eigenstates of the resonance pair (2) in terms of $p_{1}$ and $p_{2}$. Since the states 1 and 2 do not overlap, one simply obtains:

$$
p_{+}=\cos ^{4} \theta p_{1}+\sin ^{4} \theta p_{2}, \quad p_{-}=\sin ^{4} \theta p_{1}+\cos ^{4} \theta p_{2} .
$$

This change of the participation ratios must be considered together with the transformation of the parameters $a_{1}, a_{2}$ given by Eq. (7). The resulting RG equation reads:

$$
\begin{aligned}
\frac{\partial}{\partial t} f(a, p)= & 2 A_{\mathrm{d}} \nu n \int d \tau d^{n} a_{1} d^{n} a_{2} d p_{1} d p_{2}\left|\vec{a}_{1} \cdot \vec{a}_{2}\right| f\left(a_{1}, p_{1}\right) f\left(a_{2}, p_{2}\right) \\
\times & {\left[\delta\left(a-a_{+}\right) \delta\left(p-p_{+}\right)+\delta\left(a-a_{-}\right) \delta\left(p-p_{-}\right)\right.} \\
& \left.-\delta\left(a-a_{1}\right) \delta\left(p-p_{1}\right)-\delta\left(a-a_{2}\right) \delta\left(p-p_{2}\right)\right] .
\end{aligned}
$$

Generally, one cannot factor the distribution $f(a, p)$, because the flow (13) generates nontrivial correlations between $\vec{a}$ and $p$.

It is of interest to consider the flow (13) in the scaling limit, when the distribution of $\vec{a}$ given by $\int f(a, p) d p$ evolves to the fix points discussed in Sec. 11. We studied numerically evolution of the participation ratios distribution $\int f(a, p) d^{n} a$.

Because of the expected power law scaling $p \sim L^{-\mu}=\exp (-\mu t)$ it is natural to consider the distribution of $\ln p$. The results of the simulation for $n=2$ and $n=1$ are shown in Figures 3,4. Parameters used are the same as in Figures 1,2.2.

The results for $n=2$ and $n=1$ are quite different. For $n=2$, the distribution of $\ln p$ forms a peak of slowly varying width. The center of the distribution moves linearly with the RG time $t$, which corresponds to a single scaling exponent $\mu$ given by the peak velocity. In contrast, for $n=1$, the left edge of the distribution is fixed at $\ln p=0$. After rescaling, the distributions at different $t$ collapse on one another. This means that participation ratios are characterized by a continuous spectrum of scaling exponents $\mu$, rather than by a single exponent.

The values of $\mu$ are not universal because of a many-parameter family of fix points parameterized by $G_{\alpha \beta}$ as discussed in Sec. 11. Even for spherically symmetric distributions with $G_{\alpha \beta}=G \delta_{\alpha \beta}$, the distribution depends on the dimensionless parameter $\lambda=G n \nu$. The dependence on $\lambda$ however is quite simple. Numerically, we find that doubling $\lambda$ doubles velocity of the center of the distribution of $\ln p$, without changing 


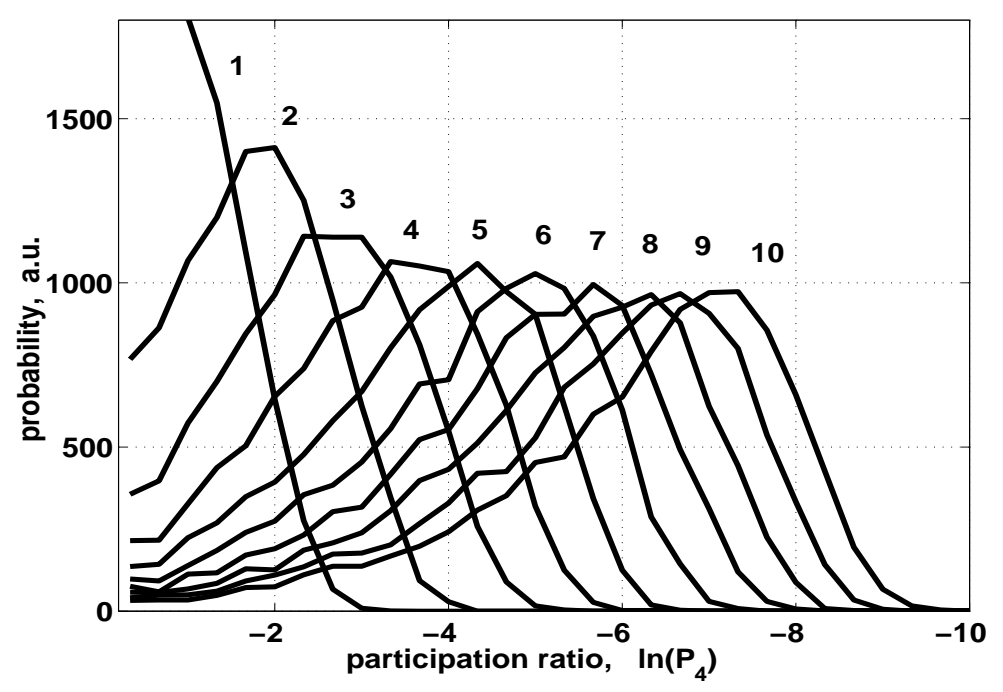

Fig. 3 The flow of participation ratios for $n=2$. All parameters are the same as in Figure 1. Distributions are numbered according to the RG times $t_{m}=2 m T, 1 \leq m \leq 10$.

of its overall form. This implies that scaling exponents $\mu$ can be written as $\gamma \lambda$, where $\gamma \simeq 1$ and has a universal distribution.

This estimate is in agreement with the result for the mean participation ratio [3], where it was found that $\langle p\rangle$ scales as $L^{-\gamma \lambda}$ with $\gamma \simeq 1$.

\section{$3 \quad$ RG for fermionic Hamiltonians}

In some cases, the RG approach outlined above can be extended to many-body problems [9]. Here we consider a Hamiltonian for interacting two-level systems in $d=3$ :

$$
\mathcal{H}=\sum_{i} \epsilon_{i} \sigma_{i}^{z}+\frac{1}{2} \sum_{i \neq j} r_{i j}^{-3} V_{i j}^{a b} \sigma_{i}^{a} \sigma_{j}^{b}
$$

Here $\sigma_{i}^{a}$ are pseudospin Pauli matrices $(a, b=x, y, z)$, and $r_{i j}=\left|r_{i}-r_{j}\right|$ are distances between two-level systems randomly distributed in space with concentration $n$. Both $\epsilon_{i}$ and $V_{i j}^{a b}$ are random uncorrelated numbers.

The problem (14) describes several physical situations, such as the dynamics of excitations in two-level systems in glasses interacting via elastic strain [8], or dipole excitations in the Efros-Shklovskii model of a disordered Mott insulator [7]. In the latter problem, the conventional approach is to consider only $\sigma_{i}^{z} \sigma_{j}^{z}$ couplings in (14). In this case excitations are strictly localized, and the only effect of interaction is the appearance of a logarithmic gap in the density of states at low energies.

Here we focus on the opposite limit, and consider the effect of $x-x, y-y$, and $x-y$ couplings, ignoring other couplings. We assume that the interaction is weak, $\lambda=\left\langle\left|V_{i j}^{a b}\right|\right\rangle n /\left\langle\left|\epsilon_{i}\right|\right\rangle \ll 1$, and develop an RG scheme using this weak coupling. The 


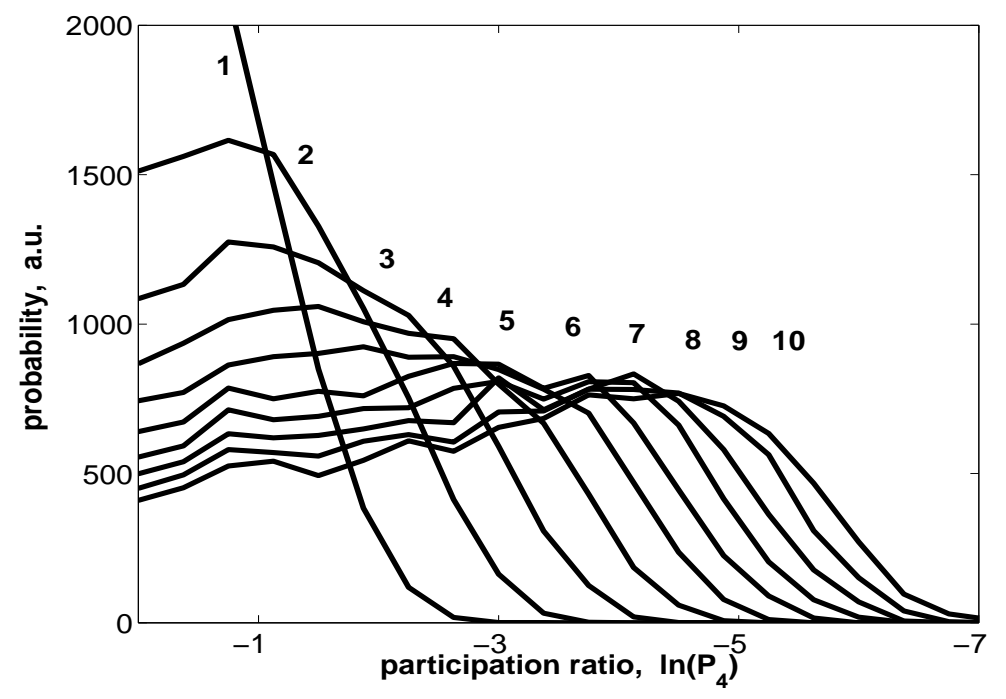

Fig. 4 The flow of participation ratios for $n=1$ corresponding to the parameters used in Figure 2. The RG times $t_{m}=2 m T, 1 \leq m \leq 10$.

$X Y$ approximation is frequently used for studying spin waves in spin systems. Although it does not seem to be controlled by any small parameter, one should mention that the terms removed have zero matrix elements between different states with close energy. Therefore, these terms cannot produce resonances (which are responsible for delocalization), so they are unlikely to be important as long as delocalization of states is concerned.

Fermionic model is obtained by replacing Pauli matrices in (14) by Fermi operators: $\sigma^{x} \rightarrow a+a^{+}, \sigma^{y} \rightarrow i\left(a-a^{+}\right), \sigma^{z} \rightarrow a^{+} a-a a^{+}$. Our main reason to employ this spinpseudofermion transformation is that it gives an exact result for a pair of interacting spins, whereas only interacting pairs contribute to the leading logarithmic order in the RG. Thus we obtain

$$
\mathcal{H}=\sum_{i} \epsilon_{i}\left(a_{i}^{+} a_{i}-a_{i} a_{i}^{+}\right)+\frac{1}{2} \sum_{i \neq j} r_{i j}^{-3}\left(u_{i j} a_{i}^{+} a_{j}+v_{i j} a_{i} a_{j}+\text { H.c. }\right)
$$

where $u_{i j}=V_{i j}^{x x}+V_{i j}^{y y}+i\left(V_{i j}^{x y}-V_{i j}^{y x}\right), v_{i j}=V_{i j}^{x x}-V_{i j}^{y y}-i\left(V_{i j}^{x y}+V_{i j}^{y x}\right)$. Distributions of real $\epsilon_{i}$ and complex $u_{i j}, v_{i j}$ are assumed to be uncorrelated. We denote them by $\nu(\epsilon)$ and $f(u, v)$, respectively: $d P=\nu(\epsilon) d \epsilon$ and $d P=f(u, v) d^{2} u d^{2} v$. The Hamiltonian (15) is bilinear in $a, a^{+}$and can be treated perturbatively by an RG in the weak coupling regime $\langle|u|\rangle \nu n \ll 1,\langle|v|\rangle \nu n \ll 1$.

The RG scheme is based on resonance pairs of pseudofermions:

$$
\mathcal{H}=\epsilon_{1}\left(a_{1}^{+} a_{1}-a_{1} a_{1}^{+}\right)+\epsilon_{2}\left(a_{2}^{+} a_{2}-a_{2} a_{2}^{+}\right)+r_{12}^{-3}\left(u a_{1}^{+} a_{2}+v a_{1} a_{2}+\text { H.c. }\right)
$$


The Hamiltonian (16) can be diagonalized by a two step Bogoliubov transformation: (1) $\left(\tilde{a}_{1}, \tilde{a}_{2}\right)=R_{\alpha}\left(a_{1}, a_{2}\right) ;(2)\left(a_{1}^{\prime+}, a_{2}^{\prime}\right)=R_{\beta}\left(\tilde{a}_{1}^{+}, \tilde{a}_{2}\right)$, where

$$
\begin{aligned}
& R_{\alpha}=\left(\begin{array}{cc}
\cos \alpha & z_{1} \sin \alpha \\
-\sin \alpha & z_{1} \cos \alpha
\end{array}\right), \quad R_{\beta}=\left(\begin{array}{cc}
\cos \beta & z_{2} \sin \beta \\
-\sin \beta & z_{2} \cos \beta
\end{array}\right), \\
& \tan 2 \alpha=\frac{r_{12}^{-3} u}{\epsilon_{1}-\epsilon_{2}}, \quad z_{1}=\frac{u}{|u|}, \quad \tan 2 \beta=-\frac{r_{12}^{-3} v}{\epsilon_{1}+\epsilon_{2}}, \quad z_{2}=\frac{v}{|v|} .
\end{aligned}
$$

The resulting diagonal Hamiltonian is $\mathcal{H}=\epsilon_{1}^{\prime}\left(a_{1}^{\prime+} a_{1}^{\prime}-a_{1}^{\prime} a_{1}^{\prime+}\right)+\epsilon_{2}^{\prime}\left(a_{2}^{\prime+} a_{2}^{\prime}-a_{2}^{\prime} a_{2}^{\prime+}\right)$, where

$$
\left(\epsilon_{1}^{\prime}-\epsilon_{2}^{\prime}\right)^{2}=\left(\epsilon_{1}-\epsilon_{2}\right)^{2}+\left|u r_{12}^{-3}\right|^{2}, \quad\left(\epsilon_{1}^{\prime}+\epsilon_{2}^{\prime}\right)^{2}=\left(\epsilon_{1}+\epsilon_{2}\right)^{2}+\left|v r_{12}^{-3}\right|^{2} .
$$

For another two-level system (say, described by $b$ and $b^{+}$) the interaction with $a_{1}$ and $a_{2}$ is given by

$$
\mathcal{H}_{\text {int }}=r_{1 b}^{-3}\left(u_{1} a_{1}^{+} b+v_{1} a_{1} b+H . c .\right)+r_{2 b}^{-3}\left(u_{2} a_{2}^{+} b+v_{2} a_{2} b+H . c .\right)
$$

In accordance with the picture of resonance pairs we can consider only the case $r_{1 b} \gg r_{12}, r_{2 b} \gg r_{12}$. Therefore, $r_{1 b}$ is almost equal to $r_{2 b}$ and hence the two Bogoliubov transformations act on $u_{1,2}$ and $v_{1,2}$ as

$$
\begin{array}{ll}
\left(\tilde{u}_{1}, \tilde{u}_{2}\right)=R_{\alpha}\left(u_{1}, u_{2}\right), & \left(\tilde{v}_{1}, \tilde{v}_{2}\right)=R_{\alpha}^{*}\left(v_{1}, v_{2}\right) ; \\
\left(u_{1}^{\prime}, v_{2}^{\prime}\right)=R_{\beta}^{*}\left(\tilde{u}_{1}, \tilde{v}_{2}\right), \quad\left(v_{1}^{\prime}, u_{2}^{\prime}\right)=R_{\beta}\left(\tilde{v}_{1}, \tilde{u}_{2}\right),
\end{array}
$$

where $*$ stands for complex conjugate. Similarly to how it was done in Sec.s 1.2, these expressions can be used to derive an RG flow for distributions:

$$
\frac{\partial}{\partial t} g(\xi)=2 \pi n e^{3 t}\left\langle\delta\left(\xi-\xi_{1}^{\prime}\right)+\delta\left(\xi-\xi_{2}^{\prime}\right)-\delta\left(\xi-\xi_{1}\right)-\delta\left(\xi-\xi_{2}\right)\right\rangle_{u, v, \xi_{1}, \xi_{2}},
$$

Here we introduced a condensed notation $\xi_{i}=\left(u_{i}, v_{i}, \epsilon_{i}\right)$, so $g(\xi)$ is a distribution of $u, v$, and $\epsilon$, the $\delta$-function $\delta\left(\xi-\xi_{1}\right)=\delta\left(u-u_{1}\right) \delta\left(v-v_{1}\right) \delta\left(\epsilon-\epsilon_{1}\right)$, etc. The brackets $\langle\ldots\rangle_{u, v, \xi_{1}, \xi_{2}}$ stand for averaging $\int \ldots f(u, v) d^{2} u d^{2} v g\left(\xi_{1}\right) d^{5} \xi_{1} g\left(\xi_{2}\right) d^{5} \xi_{2}$. (Here the integration volume elements are 2- and 5-dimensional because $u$ and $v$ are complex.) The expression $\langle\ldots\rangle_{u, v, \xi_{1}, \xi_{2}}$ in (22) is of order of $e^{-3 t}$, so the RHS of (22) has no explicit dependence on $t$.

The RG flow (22) restricted to the distribution of couplings $u, v$ is analogous to that discuss in Sec. 1. One can prove an H-theorem, verify that second moments are conserved, and derive stationary fix-point distributions:

$$
f_{w, z}(u, v)=\pi^{-2}\left(w^{2}-|z|^{2}\right) \exp \left(-w\left(|u|^{2}+|v|^{2}\right)-(z u v+\text { c.c. })\right)
$$

where $w$ is real and $z$ is complex, satisfying $|z|<w$ for normalizability of $f_{w, z}(u, v)$.

A new aspect of the pseudofermion problem is a nontrivial RG flow of the density of states. To study how the distribution of energies $\epsilon$ evolves, we consider a particular class of distributions:

$$
g(u, v, \epsilon)=\nu(\epsilon) f_{w, z}(u, v)
$$




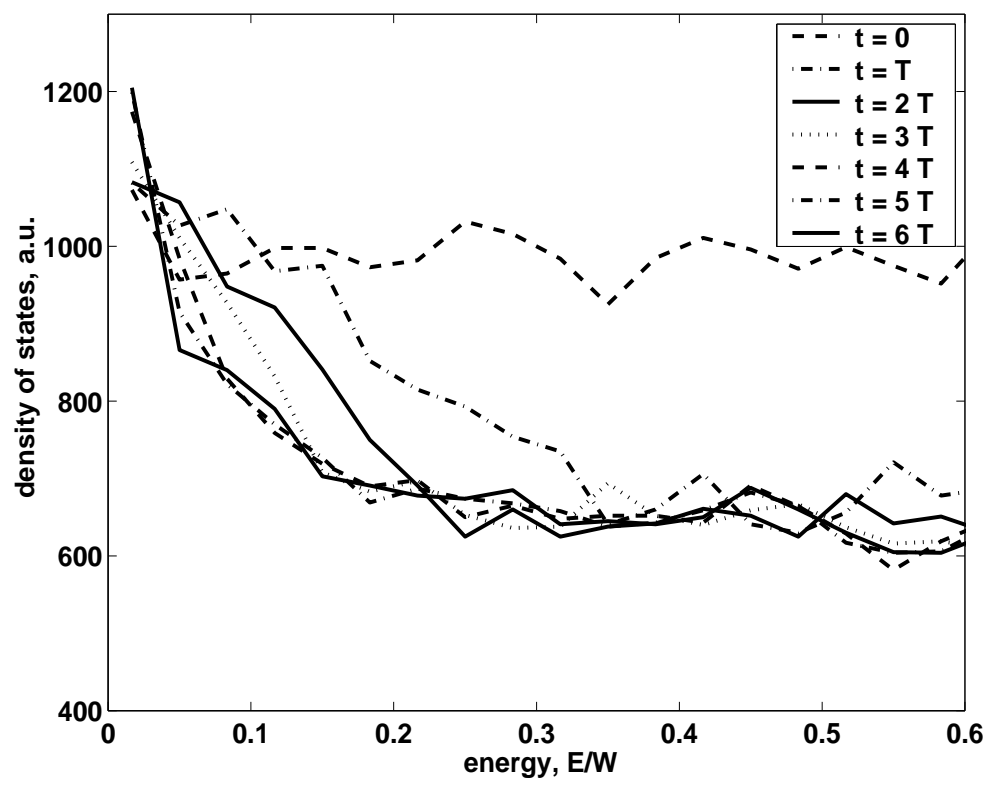

Fig. 5 The flow of the density of states for the distribution of $u, v$ of the form (23) with $\left\langle|u|^{2}\right\rangle=\left\langle|v|^{2}\right\rangle=0.45,\left\langle u v^{*}\right\rangle=0$.

with $f_{w, z}(u, v)$ being the fix-point distribution of the form (23). (Such an ansatz is consistent with Eq.(22).) Substituting this into (22) one obtains

$$
\frac{\partial}{\partial t} \nu(\epsilon)=2 \pi n e^{3 t}\left\langle\delta\left(\epsilon-\epsilon_{1}^{\prime}\right)+\delta\left(\epsilon-\epsilon_{2}^{\prime}\right)-\delta\left(\epsilon-\epsilon_{1}\right)-\delta\left(\epsilon-\epsilon_{2}\right)\right\rangle_{u, v, \epsilon_{1}, \epsilon_{2}}
$$

We studied the flow of $\nu(\epsilon)$ numerically (see Figure 5). There are two distinct features in the flow of $\nu(\epsilon)$. Initially, at small $t$, the density of states decreases at all $\epsilon$ due to energy repulsion. At large $t$, it increases in the vicinity of $\epsilon=0$ forming a singularity. It is clear from Figure 5 that the first effect is transitional and quickly saturates, whereas the second one is characteristic for the scaling limit.

It is interesting to compare this result with the Efros-Shklovskii theory of Coulomb gap which predicts a logarithmic suppression of the density of states near $\epsilon=0$ for the problem (14) with $z-z$ couplings. In the above analysis we ignored these couplings because they do not lead to delocalization of excitations. The effect of $z-z$ couplings on the density of states is of the same order as the effects we consider. However, it has an opposite sign.

Therefore, there are two different phases possible in the problem (14) depending on relative strength of different couplings. If the $z-z$ coupling dominates, the system will have a soft gap in the density of states. On the other hand, if the $x-x, y-y$, and $x-y$ couplings dominate, there will be a peak in the density of states at $\epsilon=0$. Our analysis indicates that the peak is described by a power law singularity with the exponent of order of $\lambda$. 
In both phases, however, excitations are delocalized. According to Secs. 1,2, the states in this problem are critical. Delocalized states will give rise to thermal conductivity and, in the Coulomb gap state, will make variable range hopping conductivity possible even in the absence of electron-phonon coupling.

\section{Summary}

The RG approach predicts nontrivial fix points of Hamiltonians with long range hopping. Wavefunctions in such systems are critical, and the participation ratios are characterized by scaling exponents which depend on the nature of the fix point. In some cases, for a particular Hamiltonian different states can have different exponents, and so the system is characterized by a distribution of exponents, rather than by a single exponent. (The possibility of the absence of self-averaging of the participation ratio exponent was considered recently |11].)

The RG method can be applied to the problem of interacting two-level systems. In doing this it is important to extend the RG by including renormalization of the density of states. Depending on the choice of couplings, two different phases are possible, both characterized by a singularity in the density of states at $\epsilon=0$. In one phase the density of states diverges at $\epsilon=0$, whereas in the other phase it vanishes. It is possible that the first behavior is relevant for the problem of low energy excitations in glasses, where an increase in the density of states at low energies due to interactions between two-level systems has been conjectured [10].

I benefited from discussions with B. L. Altshuler, V. E. Kravtsov, A. D. Mirlin, and B. I. Shklovskii. This research is supported in part by the MRSEC Program of NSF under award 6743000 IRG.

\section{References}

[1] P. W. Anderson, Phys. Rev. 109 (1958) 1492;

D. E. Logan, P. G. Wolynes, J. Chem. Phys. 87 (1987) 7199

[2] L. S. Levitov, Phys. Rev. Lett. 64 (1990) 547; L. S. Levitov, Europhys. Lett. 9(1) (1989) 83

[3] B. L. Altshuler, L. S. Levitov, Physics Reports 288 (1997) 487

[4] Y. V. Fyodorov, A. D. Mirlin, Phys. Rev. B52 (1995) R11580;

A. D. Mirlin, et al., Phys. Rev. E54 (1996) 3221

[5] V. E. Kravtsov, K. A. Muttalib, Phys. Rev. Lett. 79 (1997) 1913

[6] A. L. Burin and Yu. M. Kagan, Sov. Phys. JETP 82 (1996) 159

[7] A. L. Efros, B. I. Shklovskii, J. Phys. C8 (1975) L49 S. D. Baranovskii, B. I. Shklovskii and A. L. Efros, Zh. Eksp. Teor. Fiz. 78 (1980) 395 [Sov. Phys. JETP 51 (1980) 199]

[8] C. C. Yu, A. J. Leggett, Comments Cond. Mat. Phys. 14 (1988) 231 C. C. Yu, Phys. Rev. Lett. 63 (1989) 1160

[9] R. N. Bhatt, P. A. Lee, Phys. Rev. Lett. 48 (1982) 344

[10] A. L. Burin, Yu. Kagan, L. A. Maksimov, I. Ya. Polyshchuk, Phys. Rev. Lett. 80 (1998) 2945; Physica. B, Cond. Matt. 244 (1998) 180;

[11] D. A. Parshin, H. R. Schober, Distribution of fractal dimensions at the Anderson transition, preprint cond-mat/9907067 